\title{
Densidad poblacional en Chocó, Colombia, de dos árboles de importancia económica: Huberodendron patinoi (Malvaceae) e Hymenaea oblongifolia (Fabaceae)
}

\author{
Keiler Perea Pandales. ', Luz Yorleida Palacios T. ${ }^{2}$, Danilza Marcela Bellido C. ${ }^{3}$, Nohora Elia Blanquicet G4 \\ \& Jhon Tailor Rengifo Mosquera ${ }^{5}$
}

1. Grupo de Investigación de Recursos Naturales y Toxicología Ambiental, Facultad de Ciencias Naturales, Programa de Biología, Universidad Tecnológica del Chocó, "Diego Luis Córdoba”; keilerpereapandales@yahoo.es

2. Grupo de investigación de Flora Chocoana, Facultad de Ciencias Naturales, Programa de Biología, Universidad Tecnológica del Chocó “Diego Luis Córdoba"; yorleida16@hotmail.com

3. Grupo de Investigación de Ecología y Conservación de Ecosistemas Tropicales, Facultad de Ciencias Naturales, Programa de Biología, Universidad Tecnológica del Chocó “Diego Luis Córdoba”; damabeco02@gmail.com

4. Investigadora del Proyecto Aplicación de la CTel para el Mejoramiento del sector Maderero en el departamento del Chocó ejecutado por la Universidad Tecnológica del Chocó "Diego Luis Córdoba”; noeblago@hotmail.com.

5. Grupo de Investigación de Herpetología, Programa de Biología, Universidad Tecnológica del Chocó, "Diego Luis Córdoba";

jhontailorrengifo@gmail.com

Recibido 19-VII-2017 • Corregido 20-IX-2017 • Aceptado 20-X-2017

\begin{abstract}
Population density in Choco, Colombia, of two tree species of economic importance: Huberodendron patinoi (Malvaceae) and Hymenaea oblongifolia (Fabaceae). The status of two economically important tree species, Huberodendron patinoi and Hymenaea oblongifolia, in Chocó, Colombia, was unknown. We used 50x50m plots to survey seedlings and trees. We found 19 adult $\mathrm{H}$. oblongifolia; and 19 adults and 66 seedlings of $H$. patinoi (i.e. 4 Ind/ha and $33 \mathrm{lnd} / \mathrm{ha}$, respectively). Management action is needed to increase the density of both species.
\end{abstract}

RESUMEN: El estado de dos especies arbóreas de importancia económica, Huberodendron patinoi e Hymenaea oblongifolia, en Chocó, Colombia, era desconocido. Usamos parcelas de 50x50m para estudiar plántulas y árboles. Encontramos 19 H. oblongifolia adultas; y 19 adultos y 66 plántulas de H. patinoi (es decir, 4 Ind/ha y 33 Ind/ha, respectivamente). Es necesario ejecutar acciones de manejo para aumentar la densidad de ambas especies.

Palaras claves: Dinámica, especies forestales, población

Key words: Dynamics, forest species, population

Del bosque se extraen muchos productos, entre ellos la madera para la construcción, otros productos forestales no maderables como: leña frutos, setas, plantas comestibles, medicinales, ornamentales entre otras. (Tapia \& Chilpa, 2008). Los bosques primarios residuales, así como los bosques secundarios, poseen una oferta importante de especies maderables de alto valor comercial. Por tanto, la determinación en la aptitud de uso de las especies forestales con fundamento en la caracterización de sus propiedades físicas y mecánicas, permite fomentar el uso de la madera (Puertas, Guevara \& Espinoza, 2013), con lo cual se espera que al incrementar su valor comercial se impulsen procesos que permitan el manejo y conservación de este importante recurso.

La madera es un recurso natural cuya calidez, belleza y cualidades tecnológicas se suman al hecho de ser 
totalmente renovable, según los sistemas de gestión que la ciencia forestal viene desarrollando desde el siglo XVIII (Tolosa, González \& Vignote, 2004). En la actualidad, el ser humano explota aproximadamente 3400 millones de $\mathrm{m}^{3}$ de madera, de los cuales una mitad se consume en el lugar de corte o extracción, fundamentalmente como combustible (leña o carbón), y la otra mitad se utiliza como madera en rollo industrial dedicada a la construcción y otros usos.

Debido a que la madera para uso industrial es el producto más relevante, la determinación y el conocimiento de las propiedades tecnológicas de la misma tienen una importancia primordial porque pueden variar según la procedencia y las condiciones de crecimiento. De este conocimiento depende en gran medida darle el uso adecuado y la posibilidad de sugerir nuevas técnicas de manejo a la madera (Borja \& Tamarit, 1997).

Según estadísticas del DANE (2002) en el Chocó colombiano las comunidades solo utilizan el $60 \%$ de los árboles extraídos del bosque como madera para procesos de industrialización y comercialización. Un 30\% lo usan como leña, carbón entre otras. Estudios previos de estas especies incluyen: FAO (1998); CIRAD, (2003). Confemaderas \& Unión Europea, 2004, Gómez et al (2007), Montañez, et al. (2010), Canales-Springett, Stuva, Domínguez y Castillo, (2013). En el Chocó propiamente: IIAP \& Codechoco (2008); Robledo-Murillo, (2009); Zuluaga, (2010); CogolloCalderón y García-Cossío, (2012); Serna et al. (2012). Sin embargo, para el Chocó, no existen datos apropiados de la densidad de estas especies, por lo que aquí presentamos los resultados de un muestreo reciente.

\section{MATERIALES Y MÉTODOS}

La zona objeto de estudio está ubicada en el departamento del Chocó, región del Pacífico en el noroeste del

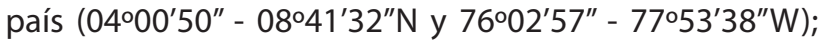
comprende las selvas del Darién y las cuencas de los ríos Atrato, San Juan y Baudó, siendo su capital la ciudad de Quibdó; este es considerado el lugar con la mayor pluviosidad del planeta; presentando una extensión de $46530 \mathrm{~km}^{2}$, que corresponden al $4 \%$ de la extensión del país.

Características de la zona de muestreo: La presente investigación se llevó a cabo en la Estación Ambiental del Alto San Juan, en la localidad de Angostura, la cual está ubicada en la parte alta del río San Juan; esta zona se localiza en el municipio de Tadó el cual se encuentra ubicado en el departamento del Chocó $\left(76^{\circ} 73^{\prime} 10^{\prime \prime} \mathrm{N}\right.$ - 5016'10"W); posee una altura de $75 \mathrm{msnm}$ y está localizado a una distancia de $68 \mathrm{Km}$, de la capital del departamento (Quibdó). Para la zona objeto de estudio Los bosques se encontraron con alta intervención antrópica contrario a lo que se dice que es uno de los ecosistemas de mayor biodiversidad en el Chocó EOT (2000 - 2009). Para la ejecución del objetivo en campo se utilizó la metodología propuesta por Lamprecht (1990) con algunas modificaciones, para cada una de las especies se estableció una parcela de 50×50m, donde se registraron en forma de zig zag todos los individuos con DAP $\geq 10 \mathrm{~cm}$ (adultos). A partir del árbol madre se midieron $15 \mathrm{~m}$ de radio $\left(707 \mathrm{~m}^{2}\right)$ donde se estableció una parcela circular en la cual se registraron todos los individuos mayores a $1,3 \mathrm{~m}$ de altura y con DAP $\leq 10 \mathrm{~cm}$ (juveniles); siguiendo las diagonales de la primera parcela se trazaron 12 cuadriculas de $2 \mathrm{~m}\left(4 \mathrm{~m}^{2}\right)$ donde se censaron todos los individuos con altura entre 0,3 y 1,3 m (plántulas). Debido al peligro de artefactos explosivos en la zona, solo se tomó la información de dos especies, Hymenaea oblongifolia y Huberodendron patinoi. Esta investigación se ejecutó con aprobación del Consejo Comunitario Mayor del Alto San Juan (ASOCASAN) y el consejo comunitario local.

\section{RESULTADOS}

Composición florística: Se registraron 85 individuos pertenecientes a dos familias con su estado conservación. Dos géneros y dos especies respetivamente. (Huberodendron patinoi y Hymenaea oblongifolia) (Cuadro 1).

\section{CUADRO 1}

Listado de especies, gremios ecológicos y estados de conservación encontradas en el municipio de Tadó, departamento del Chocó-Colombia.

\begin{tabular}{lccccccc}
\multicolumn{1}{c}{ Familias } & Nombre Común & Nombre Científico & $\begin{array}{c}\text { Gremios } \\
\text { Ecológicos }\end{array}$ & $\begin{array}{c}\text { Estado de } \\
\text { conservación }\end{array}$ & Plántulas & Juveniles & Adultos \\
Malváceae & Carrá & Huberodendrun patinoi & Heliófitas & Vulnerable (VU) & 0 & 0 & 10 \\
Fabaceae & Algarrobo & Hymenaea oblongifolia & Heliófitas & Casi amenazado (NT) & 66 & 0 & 9 \\
Total & & & & & 66 & 0 \\
\hline
\end{tabular}


CUADRO 2

Regeneración natural de las especies presentes en el municipio de Tadó, departamento del Chocó-Colombia.

\begin{tabular}{|c|c|c|c|c|c|c|c|c|c|c|c|}
\hline Especie & Plántula & Brinzal & Total & $\begin{array}{c}\text { Área } \\
\text { muestreada } \\
\text { (ha) }\end{array}$ & $\begin{array}{c}\text { Densidad } \\
\text { (ind/ha) }\end{array}$ & Latizal & $\begin{array}{c}\text { Área } \\
\text { muestreada } \\
\text { (ha) }\end{array}$ & $\begin{array}{c}\text { Densidad } \\
\text { (ind/ha) }\end{array}$ & Fustal & $\begin{array}{c}\text { Área } \\
\text { muestreada } \\
\text { (ha) }\end{array}$ & $\begin{array}{c}\text { Densidad } \\
\text { (ind/ha) }\end{array}$ \\
\hline H.patinoi & 0 & 0 & 0 & 0,048 & 0 & 0 & 0,707 & 0 & 10 & 2,5 & 4 \\
\hline H. oblongifolia & 98 & 40 & 138 & 0,048 & 2875 & 0 & 0,707 & 0 & 9 & 2,5 & 3,6 \\
\hline
\end{tabular}

De los 85 individuos 66 son plántulas (Huberodendron patinoi) y 19 son adultos (Hymenaea oblongifolia). Cabe resaltar que los individuos de la especie $H$. oblongifolia se encontraron a cuatro horas de camino y los individuos de la especie H. patinoi se encontraron en la zona llamada Yerrecui, con intervención antrópica.

De la especie Huberodendron patinoi solo se encontraron individuos en estado Fustal, mientras que Hymenaea oblongifolia registró individuos plántulas; Brinzal y Fustal. (Cuadro 2). Sin embargo, esta cantidad de plántulas que presentó $H$. oblongifolia podría disminuir drásticamente, se encontraron una cantidad de individuos con hongos, en muy mal estado por no recibir los nutrientes suficientes y por estar expuestas a la depredación de roedores.

Densidad poblacional: Se registró una densidad total de 33 ind/ha, la especie $H$. patinoi se encontró una densidad de 4 ind/ha y para $H$. oblongifolia se encontró un total de $65,3 \mathrm{ind} / \mathrm{ha}$.

No existen diferencias estadísticamente significativas entre especies (Fig. 1) y entre los estados sucesionales de desarrollo ( $>0,05$, Fig. 2$)$.

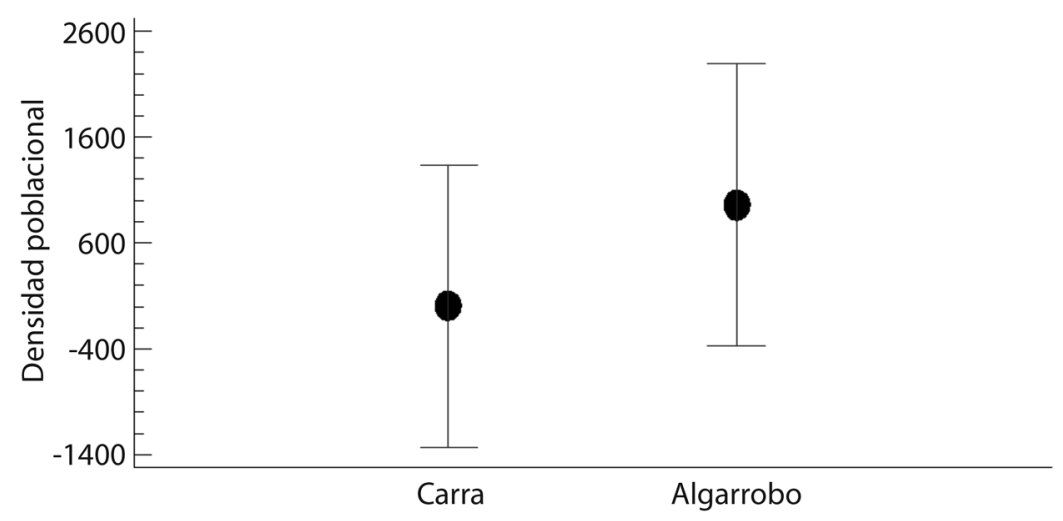

Fig. 1. Densidad poblacional por especies. Prueba $F 1,00=P$ valor $=0,3737$ los círculos son los promedios y las líneas los intervalos de confianza al 95\%.

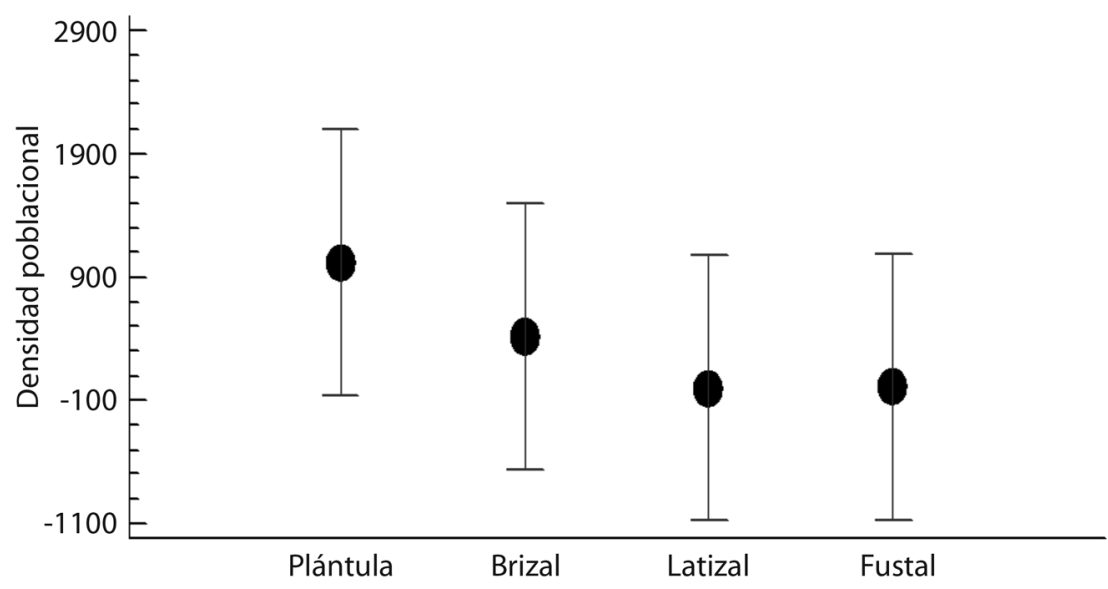

Fig. 2. Densidad poblacional por estados de desarrollo de las especies. Prueba $F=0,76$; $P$-valor $=0,5706$ los círculos son los promedios y las líneas los intervalos de confianza al 95\%. 


\section{DISCUSIÓN}

Las especies en estudios tienen gran potencial económico por su madera de gran calidad, dureza, resistencia y densidad, y por ello son altamente comercializadas en los mercados locales y nacionales. Autores como Janzen (1970) manifiesta que las plantas que crecen alrededor de los árboles madres son más susceptibles a obtener patologías, y a su vez sirven de alimentos para distintos tipos de animales. Por otro lado, las plántulas de $\mathrm{H}$. oblongifolia se encontraron cerca del árbol madre, lo que concuerda con lo afirmado por Hartshorn (1983) quien dijo que la regeneración natural es muy abundante en las cercanías del árbol madre.

Klinger, Ramírez y Guerra (2011) afirman que cerca del $40 \%$ de las poblaciones de $\mathrm{H}$. patinoi, han sido diezmadas gracias a la intensa explotación de este recurso. Caso similar le ocurre a la especie $\mathrm{H}$. oblongifolia quien es muy apetecida por los comercios, según lo manifiesta el consejo comunitario de esta zona. Advertimos la situación de agotamiento que hoy presenta esta esta especie.

Estas especies son unas de las fuentes de ingreso más importante en la economía del corregimiento de Angostura, por lo que este tipo de información urge para desarrollar planes de manejo para su uso sostenible.

\section{AGRADECIMIENTOS}

Esta investigación fue financiada con recursos de regalías (Gobernación del Chocó) y ejecutada por la Universidad Tecnológica del Chocó "Diego Luis Córdoba" en el marco del proyecto "Aplicación de la CTel para el Mejoramiento del sector Maderero en el departamento del Chocó". Bajo la dirección general de Yesid Emilio Aguilar Lemus, Yan Arley Palacios Ramos quien agradecen a la comunidad de Tadó (Angostura - Yerrecui) la hospitalidad y colaboración de los habitantes de esta zona.

\section{REFERENCIAS}

Borja de la R., A., \& Tamarit U., J. C. (1997). Propiedades tecnológicas de la madera de Pinus Arizona Engelm del estado de Durango, México. Revista Chapingo Serie Ciencias Forestales \& Ambiente, 3(1), 103-108.

Canales-Springett, A. W., Stuva, A. C., Domínguez, G., \& Castillo, A. (2013). Respuesta de la regeneración natural de la Uncaria tomentosa (willd) d.c. "uña de gato", al efecto de la luz en ecosistemas boscosos primarios intervenidos dentro del bosque nacional Alexander Von Humboldt, pucallpa - Perú. Ecología Aplicada, 12(2), 99-109. doi: 10.21704/rea.v12i1-2.443

CIRAD. (2003). Jatoba. Guía de las diferentes Maderas y de su Secado. Recuperado de www. marinebox.com.br

Cogollo-Calderón, A. M, \& García-Cossío, F. (2012). Caracterización etnobotánica de los productos forestales no maderables (PFNM) en el corregimiento de Doña Josefa, Chocó, Colombia. Revista Biodiversidad Neotropical, 2(2), 102-112. doi: 10.18636/bioneotropical. v2i 2.70

Confemaderas, \& Unión Europea. (2004). Guía práctica de especies de madera. Confederación Española de Empresarios de la Madera. C/ Sagasta, No. 24, (3a) Ficha 28004

DANE, Departamento Administrativo De Estadística Nacional. (2002). Informe de Coyuntura Económica Regional del Chocó. Bogotá, Colombia.

Gómez Restrepo, M. L., \& Toro Murillo, J. L (2007) Manejo de las Semillas y la Propagación de Diez Especies Forestales del Bosque Húmedo Tropical. Boletín Técnico Biodiversidad, 2,71 .

Hartshorn, G. S. (1983) Plants. In D. H., Janzen (ed) Costa Rica Natural History (pp. 118-157). Chicago. EE.UU. University press.

Janzen, D. H. (1970). Herbivores and the number of tree species in the tropics. The American Naturalist, 104(940), 501-528. doi: $10.1086 / 282687$

Klinger, W., Ramírez, G., Guerra, J. M. (2011). Aportes al conocimiento de los ecosistemas estratégicos y las especies de interés especial del Chocó Biogeográfico. Parte I. Quibdó: Instituto de Investigaciones Ambientales del Pacífico.

FAO. La Organización de las Naciones Unidas para la Agricultura y la Alimentación. (1998). Serie Técnica XII. Información técnica para el procesamiento industrial de 134 especies maderables de Bolivia. Santa Cruz, Bolivia: Proyecto FAOGCP/ BOL/028/NET.

Lamprecht, H. (1990). Silvicultura en los trópicos: los ecosistemas forestales en los bosques Tropicales y sus especies arbóreas-posibilidades y métodos para un aprovechamiento sostenido. Eschborn, Alemania: (GTZ) GMBH.

Montañez, R. A., Escudero, C. Y., \& Duque, Á. J. (2010). Patrones de distribución espacial de especies Arbóreas en bosques de alta montaña del departamento de Antioquia, Colombia. Revista Facultad Nacional de Agronomía, Medellín 63(2), 5629-5638.

Puertas, P, Guevara, C., \& Espinoza, M. (2013). Utilización industrial y mercado de diez especies maderables potenciales de bosques secundarios y primarios residuales. Lima, Perú: Asociación para la Investigación y Desarrollo integral. Revista. Biodiversidad. Neotropical; 2(2), 102-12.

Robledo-Murillo, D. (2009). Implementación de estudio base para especies forestales amenazadas, en el municipio 
de Alto Baudó, departamento del Chocó, Colombia. Revista Bioétnia, 6(2), 82-92 pp.

Serna Mosquera, Y. B., Borja de la Rosa, A., Fuentes Salinas, M., \& Corona Ambriz, A. (2011). Propiedades tecnológicas de la madera de algarrobo (Hymenaea oblongifolia Huber), de Bagadó-Chocó, Colombia. Revista Chapingo serie ciencias forestales y del ambiente, 17(3), 411-422. doi: 10.5154/r.rchscfa.2010.07.043

Tapia-Tapia, E. C., \& R. Reyes Chilpa. (2008). Productos forestales no maderables en México: aspectos económicos para el desarrollo sustentable. Madera y Bosques, 14(3) ,95-112. doi: $10.21829 /$ myb.2008.1431208

Tolosa E., González V. M., \& Vignote S. (2004). El Aprovechamiento Maderero. 2a Ed. Madrid, España: Mundi-Prensa.

Zuluaga Ramirez, S. Regeneracion inicial y composicion de formas de vida de plantas en rozas experimentales en una selva humeda tropical del chocó colombiano. Ecotropicos, 23, 2. 